\title{
Protein Prune Homolog 2
}

National Cancer Institute

\section{Source}

National Cancer Institute. Protein Prune Homolog 2. NCI Thesaurus. Code C104806.

Protein prune homolog 2 (3088 aa, $\sim 341 \mathrm{kDa}$ ) is encoded by the human PRUNE2 gene.

This protein may be involved in the regulation of apoptosis. 